\title{
Láser erbium vaginal como tratamiento del síndrome genitourinario de la menopausia: resultados preliminares
}

\author{
Marco Levancini A. ${ }^{1,2,3}$, Marco Gambacciani. ${ }^{1}$ \\ ${ }^{1}$ Departamento de Obstetricia y Ginecología, Hospital Universitario Santa Chiara, Pisa. Italia. ${ }^{2}$ Departamento de Obs- \\ tetricia y Ginecología, Clínica Alemana. Santiago. Chile. 3Unidad de Gestión Clínica de la Mujer y el Recién Nacido, \\ Hospital Padre Hurtado. Facultad de Medicina Clínica Alemana-Universidad del Desarrollo, Santiago. Chile.
}

\section{RESUMEN}

Antecedentes: El síndrome genitourinario de la menopausia (SGM) afecta hasta el 50\% de las mujeres posmenopáusicas. Sus síntomas presentan un curso crónico y progresivo, pudiendo alterar significativamente la sexualidad y la calidad de vida. Objetivo: Evaluar efectividad a corto plazo y aceptabilidad del láser vaginal de Erbium en cristal de itrio-aluminio-granate (Er:YAG) como una terapia fototérmica no invasiva, no ablativa para el manejo del SGM. Método: Estudio longitudinal prospectivo, realizado en 28 mujeres posmenopáusicas con SGM. Se aplica láser vaginal Er:YAG en 3 sesiones cada 30 días. En todos los controles, se evalúa sequedad vaginal, dispareunia mediante escala visual analógica, y se registra el "vaginal health index score" (VHIS). Al final del tratamiento se evalúa la aceptabilidad del método. Resultados: Se observa mejoría significativa de los síntomas (sequedad vaginal y dispareunia; $p<0,01$ ) durante todo el periodo de estudio y del VHIS $(8,2 \pm 1,3$ previo al tratamiento vs. $19,2 \pm 0,4$ posterior al tratamiento; p<0,01). El 93,4\% de las pacientes calificó el procedimiento como excelente o bueno. Conclusión: Nuestro estudio piloto muestra que el tratamiento con láser Er:YAG en mujeres posmenopáusicas con síntomas de SGM es eficaz, factible y seguro en el corto plazo. Se requieren datos a más largo plazo y de mayor tamaño muestral para confirmar nuestros resultados.

\section{PALABRAS CLAVE: Menopausia, estrógenos, láser Erbium, atrofia vulvovaginal, síndrome genitourinario de la menopausia}

\section{SUMMARY}

Background: The genitourinary syndrome of menopause (GSM) affects up to $50 \%$ of postmenopausal women. Symptoms are chronic and likely worsen over time. This complains may interfere with sexual function and quality of life. Objective: To evaluate the short-term effectiveness and acceptability of Erbium in yttrium aluminum-garnet crystal Laser (Er:YAG) as a non-invasive, non-ablative photothermal therapy for the treatment of GSM. Methods: Prospective longitudinal study performed in 28 postmenopausal women suffering from GSM. All participants were treated intravaginally with Er:YAG laser in 3 applications performed every 30 days. Symptoms (vaginal dryness and dyspareunia) were evaluated by a visual-analogic scale (VAS) and the vaginal health index score (VHIS). After therapy, the women were asked to evaluate the acceptability of the intervention. Results: Er:YAG laser treatment was effective to improve symptoms (vaginal dryness and dyspareunia; $p<0.01)$ at all the study period, as well the VHIS $(8.2 \pm 1.3$ at baseline vs. $19.2 \pm 0.4$ after treatment; $p<0.01$ ). Satisfaction with the procedure was reported as excellent or good by 93.4\% of women. Conclusion: Our pilot study shows that the Er:YAG treatment in postmenopausal women with GSM is effective, feasible, and safe. Further larger, long-term and controlled studies are required to confirm this data.

KEY WORDS: Menopause, estrogen, Erbium laser, vulvovaginal atrophy, genitourinary syndrome of menopause 


\section{INTRODUCCIÓN}

El síndrome genitourinario de la menopausia (SGM) es la denominación recomendada para agrupar los síntomas relacionados a los cambios físicos de la vulva, la vagina y el tracto urinario inferior secundarios al hipoestrogenismo (1). EI SGM puede afectar hasta el $50 \%$ de las mujeres posmenopáusicas, sus síntomas tienden a ser crónicos y progresivos (2-8), afectando la sexualidad y la calidad de vida (9-15). Algunas preparaciones, incluidos lubricantes y humectantes mejoran los síntomas, sin embargo proporcionan un alivio temporal. La terapia de reemplazo hormonal $(\mathrm{TRH})$ puede proporcionar un alivio rápido y a largo plazo (16). La administración de estrógenos vaginales locales es el tratamiento de elección $(2,3)$. Aunque no han sido identificados riesgos sistémicos con estrógenos locales de baja dosis, los datos de eficacia y seguridad a largo plazo son escasos. Además, muchas mujeres actualmente no desean TRH por tiempos prolongados, o presentan contraindicaciones, como los antecedentes personales de tumores dependientes de estrógenos, particularmente cáncer de endometrio y cáncer de mama (16). Es por esto, que desde hace un tiempo se han buscado otras alternativas terapéuticas para este grupo de mujeres.

La tecnología láser es ampliamente utilizada actualmente en áreas como dermatología, odontología, oftalmología y medicina estética con efectos positivos demostrados a nivel básico y clínico sobre la remodelación de tejidos, la colagenogénesis y la angiogénesis (17-22).

El objetivo del estudio es evaluar la eficacia y la aceptabilidad del nuevo láser de erbium en cristal de itrio-aluminio-granate (Er:YAG) como una terapia fototérmica no invasiva, no ablativa para el manejo del SGM.

\section{PACIENTES Y MÉTODO}

Estudio longitudinal prospectivo, realizado en 28 mujeres asistentes a la clínica de la menopausia del Hospital de la Universidad de Pisa (Italia) que sufren de SGM. Todas las pacientes firmaron consentimiento informado. El protocolo fue revisado por un Comité consultor independiente a nivel nacional, y fue aprobado por el Comité de Ética del Departamento de Obstetricia y Ginecología del Hospital.

Los criterios de inclusión fueron la presencia de SGM en mujeres posmenopáusicas (12 meses desde el último período menstrual u ooforectomía bilateral) con niveles plasmáticos de gonadotropina y estradiol en rangos posmenopáusicos (FSH > 40 U/L; estradiol $<25 \mathrm{pg} / \mathrm{ml}$ ) y citología cervical negativa (Papanicolaou). Los criterios de exclusión fueron: lesión o infección activa o reciente (30 días) en el tracto genitourinario, sangrado uterino anormal, uso de lubricantes o cualquier otra preparación local dentro de los 30 días anteriores a la inclusión en el estudio, historia de trastorno de fotosensibilidad o el uso de medicamentos fotosensibilizantes, prolapso genital (grado II-III clasificación POP-Q), enfermedad grave o crónica que pudiera interferir con el cumplimiento del estudio y que hayan sido tratadas con hormonas u otros medicamentos para aliviar los síntomas de la menopausia en los 3 meses anteriores.

Protocolo de estudio: Las participantes fueron tratadas con láser Er:YAG (XS Fotona Smooth ${ }^{\mathrm{TM}}$, Fotona, Ljubljana, Eslovenia) con una longitud de onda de $2940 \mathrm{~nm}$. El diámetro del haz de láser sobre el objetivo fue de $7 \mathrm{~mm}$, con pulsos según la modalidad SMOOTH'M , a una frecuencia de $1,6 \mathrm{~Hz}$, y "fluence" (energía láser emitido por unidad de área) de $6,0 \mathrm{~J} / \mathrm{cm} 2$. Los parámetros fueron seleccionados en base a estudios preclínicos y clínicos realizados en diferentes condiciones experimentales (21). La tecnología "Variable Square Pulse" (VSP) posee la cualidad de poder controlar simultáneamente la energía y el tiempo de duración (o ancho de pulso), de esta forma se logra reducir la potencia y un aumento de la duración del pulso (21). El modo SMOOTH ${ }^{\mathrm{TM}}$, distribuye el calor aproximadamente a 100 micras de profundidad de la superficie mucosa, logrando un efecto térmico profundo, controlado y sin ablación.

Los procedimientos se realizaron en forma ambulatoria, sin uso de medicamentos o anestesia antes o posterior al procedimiento. El protocolo de tratamiento consiste en 3 aplicaciones de láser cada 30 días (L1, L2, L3) con una visita 2 a 4 semanas antes del tratamiento y controles de seguimiento después de 4 y 8 semanas de la última aplicación. El procedimiento se realiza, con los parámetros del programa Renovalase 1: fluence de $5,5 \mathrm{~J} / \mathrm{cm}^{2}$, con el modo SMOOTH ${ }^{\mathrm{TM}}$ a una frecuencia de $1,6 \mathrm{~Hz}$. El tamaño del punto fue $7 \mathrm{~mm}$. Después de insertar el espéculo vaginal diseñado específicamente, la sonda (R11) se introduce en el espéculo, sin contacto directo con la mucosa vaginal. Posteriormente, se lleva a cabo la irradiación circular de la pared vaginal, con 4 pulsos dados cada $5 \mathrm{~mm}$ y se retrae la sonda en $5 \mathrm{~mm}$ cada vez (utilizando la escala graduada en la sonda) hasta llegar a la entrada del canal vaginal. Este procedimiento se repite 3 veces rotando el espéculo $45^{\circ}$ cada vez para lograr abarcar toda la superficie de tejido. Finalmente, se retira el espéculo y se cambia la sonda (PSO3), se irradia el vestíbulo y el introito con los parámetros programados (Renovalase 2) con un tamaño de punto de $7 \mathrm{~mm}$, fluence de $10 \mathrm{~J} / \mathrm{cm}^{2}$, con el modo SMOOTH a $1,6 \mathrm{~Hz}$ de frecuencia. Después del tratamiento, se recomienda a los pacientes abstención de actividad sexual durante una semana.

Mediciones y pruebas: En la primera visita se 
verifica la elegibilidad del paciente y obtiene el consentimiento informado por escrito. Se registran las características sociodemográficas y características clínicas. Los síntomas subjetivos (sequedad vaginal y dispareunia) fueron evaluados mediante una escala visual analógica (VAS) en cada visita (rango $0-10 \mathrm{~cm}$; $0=$ ausencia total del síntoma y $10 \mathrm{~cm}=$ el síntoma peor posible) y durante el examen ginecológico se calculó el "vaginal health index score" (VHIS). EI VHIS evalúa el aspecto de la mucosa vaginal (elasticidad, $\mathrm{pH}$, flujo vaginal, integridad de la mucosa y la humedad). Cada parámetro se califica de 1 a 5 , siendo atrófica una puntuación total $\leq 15$ (23).

En el control postratamiento, se evaluó la aceptabilidad y la eficacia general de la terapia calificándola como excelente, buena, aceptable, malo o inaceptable. Pacientes que salen del estudio antes de la fecha límite fueron considerados abandono.

Análisis estadístico: Se trata de un estudio piloto, el tamaño de la muestra no se basó en un cálculo estadístico. El tamaño de la muestra fue planeado para ser similar a la de los datos publicados (22). Los datos se presentan como media y desviación estándar $( \pm D E)$. Las variables continuas se analizaron mediante la prueba $\mathrm{T}$ pareada y la prueba de signo-rango de Wilcoxon de acuerdo a la distribución de datos. Los datos fueron analizados utilizando el software Sigma Stat versión 3.5 y el software SPSS versión 13.0 (SPSS Science, Chicago, IL, EE.UU.). Se consideró como significativo un valor $\mathrm{p}<0,01$.

\section{RESULTADOS}

En la Tabla I se muestran datos sociodemográficos de las pacientes tratadas. Se incluyeron 27 pacientes $(96,4 \%)$ que completaron el seguimiento. Una paciente abandonó el estudio por razones personales, sin referir malestar o complicación del procedimiento (3,6\%). En nuestro grupo, 4 mujeres reportaron no presentar actividad sexual durante el periodo de estudio, por lo que los resultados para dispareunia se analizaron sobre una muestra de 23 pacientes.

Los síntomas vaginales estudiados mediante la escala VAS presentaron promedios de: $8,1 \pm 1,3$; $5,1 \pm 1,4 ; 3,2 \pm 1,4 ; 3,1 \pm 1,2 ; 3,1 \pm 1,2 \mathrm{~cm}$ para sequedad vaginal en L1, L2, L3 y controles a las 4 y 8 semanas postratamiento respectivamente (Figura 1). Con respecto a dispareunia, se observaron promedios de $8,4 \pm 1,5 ; 4,5 \pm 1,2 ; 2,6 \pm 1,2 ; 2,5 \pm$ 0,9 y $2,4 \pm 0,8 \mathrm{~cm}$ para las respectivas evaluaciones descritas anteriormente (Figura 2). EI VHIS al inicio y en cada control del período de estudio mejoró significativamente $(p<0,01)$ respecto al control basal, presentando respectivamente valores de 8,2 $\pm 1,5 ; 15,7 \pm 1,9 ; 18,0 \pm 2,0 ; 19,6 \pm 1,6$ y $19,2 \pm 1,4$ (Figura 3).
Tabla I

\section{CARACTERÍSTICAS SOCIODEMOGRÁFICAS DE LAS 28 MUJERES POSMENOPÁUSICAS INCLUIDAS EN EL ESTUDIO}

\begin{tabular}{lccc}
\hline Variable & Media & $\pm \mathrm{DE}$ & Rango \\
\hline Edad & 60,3 & 9,18 & $34-75$ \\
Talla $(\mathrm{cm})$ & 163,6 & 0,057 & $154-179$ \\
Peso $(\mathrm{Kg})$ & 68 & 11,01 & $48-89$ \\
IMC & 25,3 & 3,52 & $18,7-32,3$ \\
Edad Menopausia & 49,0 & 4,0 & $31-55$ \\
(años) & & & \\
Años desde la & 12,3 & 5,1 & $2-28$ \\
menopausia & & & \\
\hline
\end{tabular}

Al finalizar el ciclo de aplicaciones de láser, 23 pacientes $(85 \%)$ calificaron el procedimiento como excelente, 2 como bueno $(7,4 \%), 2$ pacientes $(7,4 \%)$ aceptable (Figura 4$)$. No se registraron eventos adversos relacionados con el procedimiento a lo largo del período de estudio.

\section{DISCUSIÓN}

Los diferentes tipos de láser poseen características físicas que determinan efectos específicos. La absorción del haz de luz es la base del efecto de la energía láser. Esta absorción produce interacciones fotoquímicas, fototérmicas y fotomecánicas en los tejidos. En las mucosas se ha demostrado que el láser no ablativo Er:YAG estimula la producción de proteínas de matriz y fibroblastos, generando contracción de las fibras de colágeno y cambios tróficos que se manifiestan en tejidos con mayor grosor, hidratación y tensión (24-28).

Recientemente en un estudio piloto, Salvatore y cols (22) demostraron que el tratamiento con láser fraccionado microablativo de $\mathrm{CO} 2$ indujo una mejoría significativa del SGM en mujeres postmenopáusicas.

Nuestros resultados indican que el láser vaginal no ablativo Er:YAG es factible de realizar en mujeres posmenopáusicas, es bien tolerado y podría mejorar los signos y los síntomas más molestos del GSM en ellas. Una mejoría significativa ya es posible observar después de la primera aplicación de láser y este efecto se mantiene después de la tercera aplicación tanto para la sequedad vaginal y la dispareuia, como para el índice VHIS. Durante el periodo de observación no se registraron eventos adversos. Nuestros resultados concuerdan con los reportados por Gaspar y cols usando la misma energía láser Er:YAG, (29), y por Salvatore y cols (22) usando el láser de CO2. 


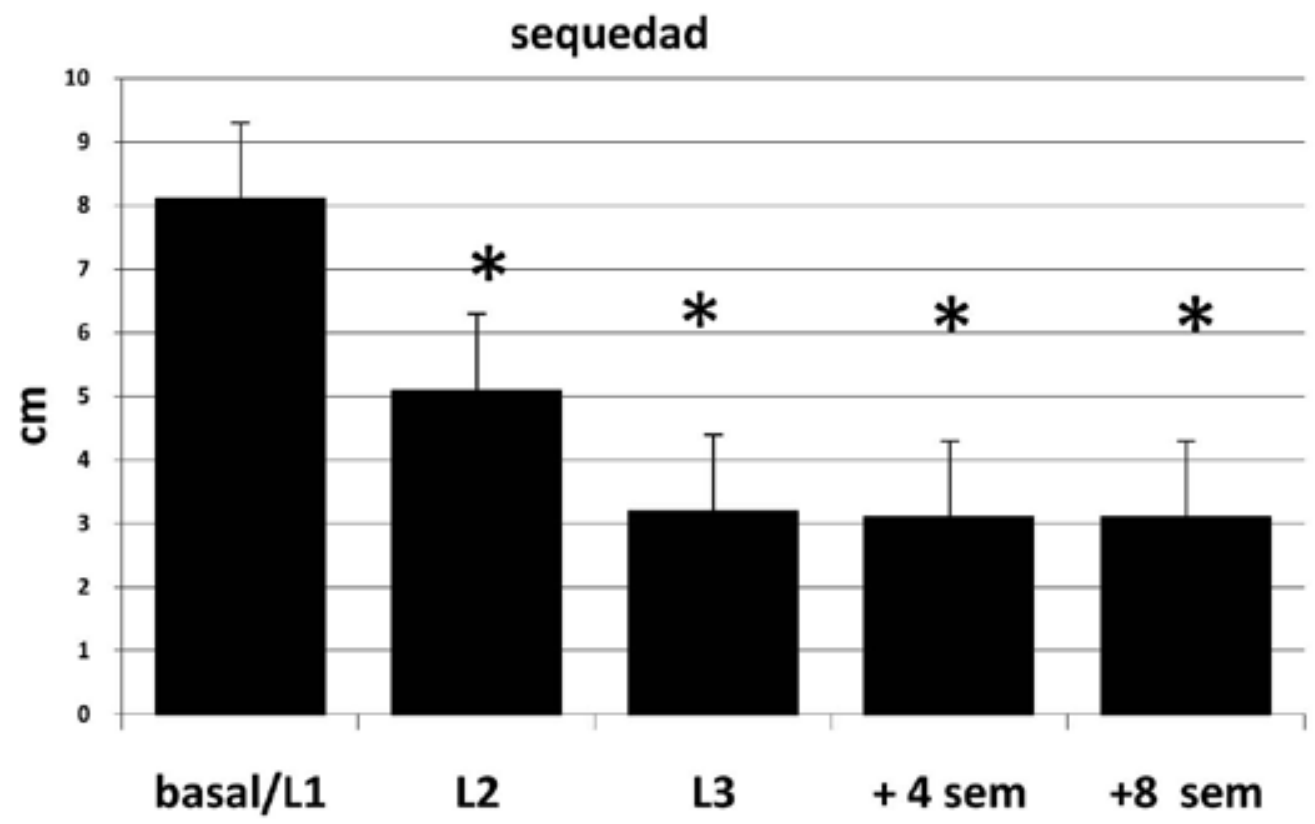

Figura 1. Escala visual análoga (VAS, cm) de sequedad vaginal, evaluada en 27 mujeres ( ${ }^{*} p<0,01$ vs. control basal).

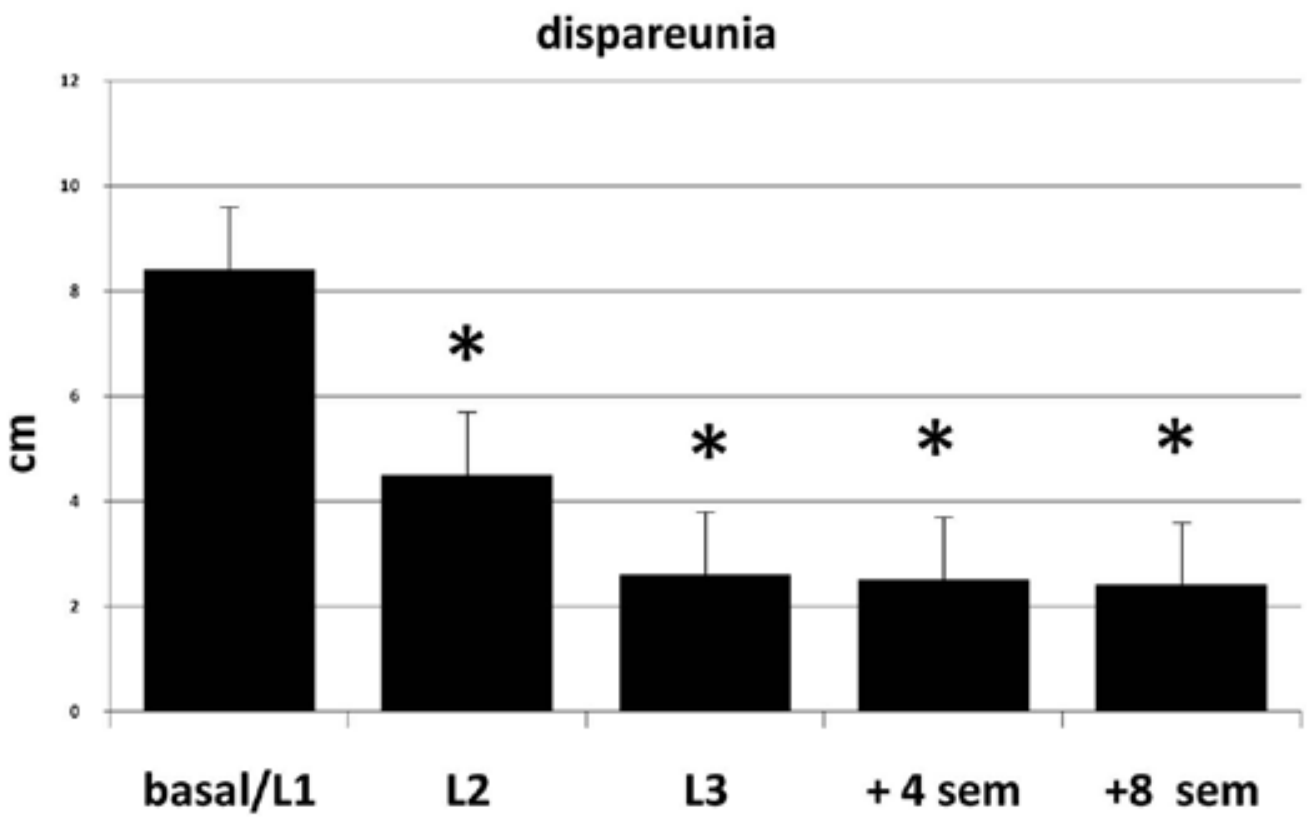

Figura 2. Dispareunia, evaluada en 23 mujeres sexualmente activas durante el periodo de estudio $\left({ }^{*} p<0,01\right.$ vs. control basal). 


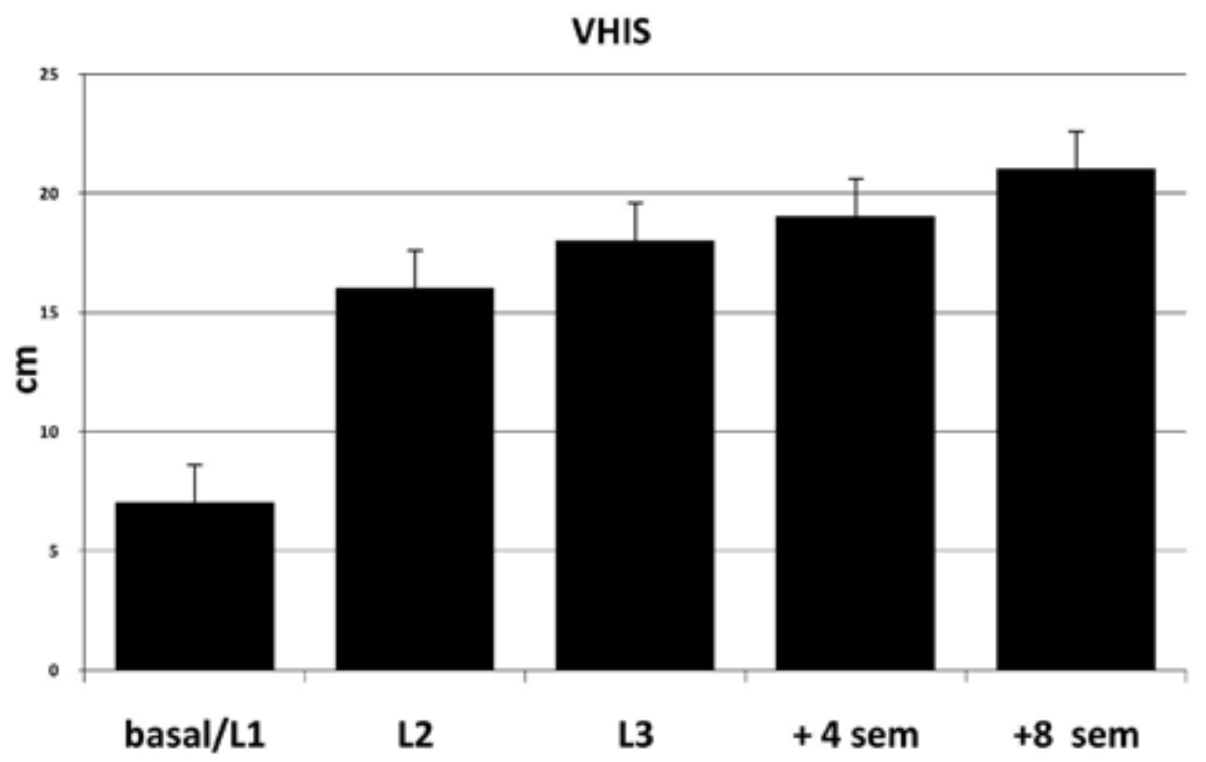

Figura 3. Evolución del VHIS desde el control basal hasta el control postratamiento en 27 mujeres portadoras de SGM. ( ${ }^{*} p<0,01$ vs. control basal).

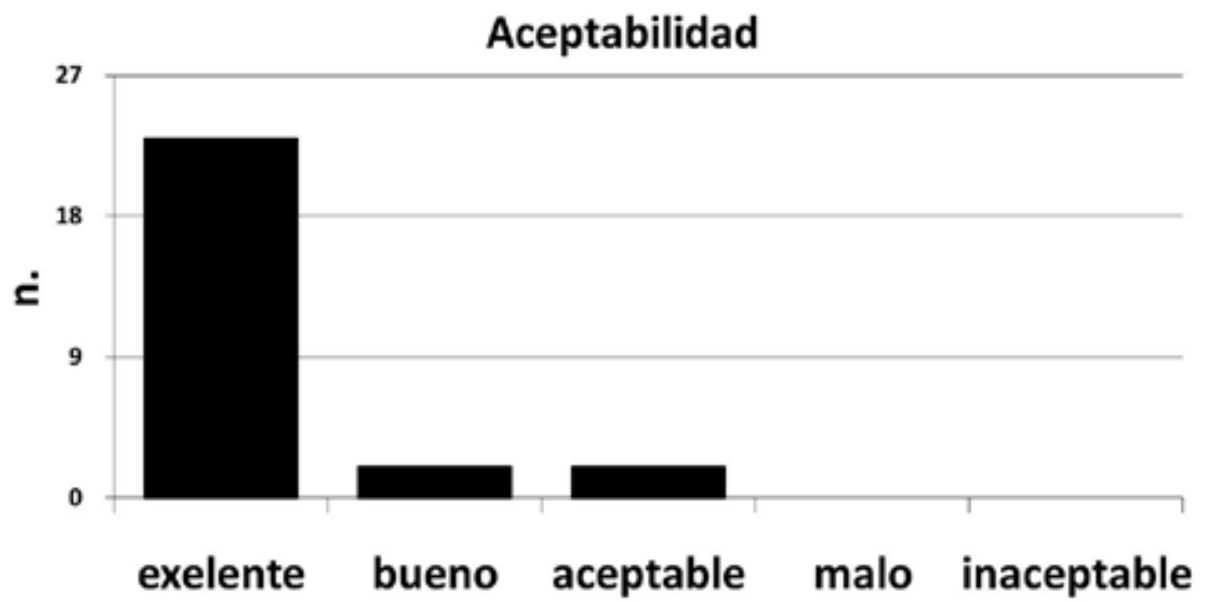

Figura 4. Aceptabilidad del láser Er:YAG al final del tratamiento en 27 mujeres portadoras de SGM .

Este estudio piloto tiene varias limitaciones: tamaño muestral pequeño, falta de seguimiento a largo plazo y la ausencia de un grupo comparador (placebo u otro tratamiento activo), sin embargo fue planeado principalmente para demostrar la factibilidad y la eficacia del procedimiento sobre los tejidos vaginales en la práctica clínica.

\section{CONCLUSIÓN}

Nuestros resultados muestran que el tratamiento con láser Er:YAG en mujeres posmenopáusicas con síntomas del SGM es factible, eficaz y seguro en el corto plazo. Si estos resultados se confirman a largo plazo y con mayor tamaño muestral, 
tendremos una opción segura y eficaz adicional para tratar el GSM y contrarrestar el envejecimiento urogenital en el manejo de la menopausia.

Agradecimientos: Los autores agradecen su excelente trabajo por revisar el presente protocolo a los miembros del Comité Consultor Independiente: Prof. Nicoletta Biglia, Torino, Prof. Rossella Nappi, Pavia, y Prof. Anna Maria Paoletti, Cagliari.

\section{REFERENCIAS}

1. Portman DJ, Gass ML; Vulvovaginal Atrophy Terminology Consensus Conference Panel. Genitourinary syndrome of menopause: new terminology for vulvovaginal atrophy from the International Society for the Study of Women's Sexual Health and The North American Menopause Society. Climateric 2014;17:557-63.

2. Sturdee DW, Panay N. Recommendations for the management of postmenopausal vaginal atrophy. Climacteric 2010;13:509-22.

3. [no authors listed]. Management of symptomatic vulvovaginal atrophy: 2013 position statement of The North American Menopause Society. Menopause 2013;20:888-902.

4. Robinson D, Toozs-Hobson P, Cardozo L. The effect of hormones on the lower urinary tract. Menopause Int 2013;19:155-62.

5. Robinson D, Cardozo L. The pathophysiology and management of postmenopausal urogenital oestrogen deficiency. J Br Menopause Soc 2001;7:67-73.

6. Ettinger $B$, Hait $H$, Reape KZ, Shu $H$. Measuring symptom relief in studies of vaginal and vulvar atrophy: the most bothersome symptom approach. Menopause 2008;15:885-9.

7. Parish SJ, Nappi RE, Krychman ML, et al. Impact of vulvovaginal health on postmenopausal women: a review of surveys on symptoms of vulvovaginal atrophy. Int J Womens Health 2013;5:437-47.

8. Oge T, Hassa H, Aydin Y, Yalcin OT, Colak E. The relationship between urogenital symptoms and climacteric complaints. Climacteric 2013;16:646-52.

9. Santen RJ, Allred DC, Ardoin SP, et al. Executive summary: Postmenopausal hormone therapy: an Endocrine Society scientific statement. J Clin Endocrinol Metab 2010;95(Suppl 1):s1-66.

10. [no authors listed]. The 2012 hormone therapy position statement of the North American Menopause Society. Menopause 2012;19:257-71.

11. de Villiers TJ, Gass ML, Haines CJ, , et al. Global Consensus Statement on Menopausal Hormone Therapy. Climateric 2013;16:203-4.

12. Blümel JE, Castelo-Branco C, Cancelo MJ, Romero $\mathrm{H}$, Aprikian D, Sarrá S. Impairment of sexual activity in middle-aged women in Chile. Menopause 2004;11:7881.

13. Monterrosa A, Blumel JE, Chedraui P, Gomez B, Valdez C. Quality of life impairment among postmenopausal women varies according to race. Gynecol Endocrinol 2009;25:491-7.
14. Figueroa JR, Jara AD, Fuenzalida PA, et al. Prevalence of sexual dysfunction among climacteric women. Rev Med Chil 2009;137:345-50.

15. Blümel JE, Chedraui $P$, Baron $G$, et al. Collaborative Group for Research of the Climacteric in Latin America (REDLINC). Sexual dysfunction in middleaged women: a multicenter Latin American study using the Female Sexual Function Index. Menopause 2009;16:1139-48.

16. de Villiers TJ, Pines A, Panay N, Gambacciani M, Archer DF, Baber RJ, Davis SR, Gompel AA, Henderson VW, Langer R, Lobo RA, Plu-Bureau G, Sturdee DW; International Menopause Society. Climateric 2013;16:316-37.

17. Kaufmann R, Hibst R. Pulsed Erbium:YAG laser ablation in cutaneous surgery. Lasers Surg Med 1996;19:324-30.

18. Reynolds N, Cawrse N, Burge T, Kenealy J. Debridement of a mixed partial and full thickness burn with an erbium:YAG laser. Burns 2003;29:183-8.

19. Lukac M, Vizintin Z, Sult T. Novel fractional treatments with variable square pulse Erbium:Yttrium-AluminumGarnet Aesthetic Lasers. Eur Dermatol 2009;4:58-61.

20. Levy JL, Trelles MA. New operative technique for treatment of xanthelasma palpebrarum: laser inverted resurfacing: preliminary report. Ann Plast Surg 2003;50:339-43.

21. Vizintin Z, Rivera M, Fistonic I, et al. Novel minimally invasive VSP Er:YAG laser treatments in gynecology. J LAHA 2012;(1):46-58.

22. Salvatore S, Nappi RE, Zerbinati N, et al. A 12-week treatment with fractional $\mathrm{CO} 2$ laser for vulvovaginal atrophy: a pilot study. Climacteric 2014;17:1-7.

23. Bachmann GA, Notelovitz M, Kelly SJ, et al. Longterm non hormonal treatment of vaginal dryness. Clin Pract Sexuality 1992;8:3-8.

24. Drnovsek-Olup B, Beltram M, Pizem J. Repetitive Er:YAG laser irradiation of human skin: a histological investigation. Lasers Surg Med 2004;35:146-51.

25. Kunzi-Rapp K, Dierickx CC, Cambier B, Drosner M. Minimally invasive skin rejuvenation with Erbium: YAG laser used in thermal mode, Lasers Surg Med 2006;38:899-907.

26. Smith KL, Skelton HG, Graham JS, et al. Increased smooth muscle actin, factor XIIla, and vimentin-positive cells in the papillary dermis of carbon dioxide laserdebrided porcine skin. Dermatol Surg 1997;23:891-5.

27. Utley DS, Koch RJ, Egbert BM. Histologic analysis of the thermal effect on epidermal and dermal structures following treatment with the superpulsed $\mathrm{CO} 2$ laser and the erbium: YAG laser: an in vivo study. Lasers Surg Med 1999;24:93-102.

28. Romeo U, Libotte F, Palaia G, et al. Histological in vitro evaluation of the effects of Er:YAG laser on oral soft tissues. Lasers Med Sci 2012;27:749-53.

29. Gaspar A. Comparison of New Minimally Invasive Er:YAG Laser Treatment and Hormonal Replacement Therapy in the treatment of Vaginal Atrophy. Climacteric 2014;17(Suppl 1):48-108, P 124. 